\title{
Front Matter: Volume 9886
}

, "Front Matter: Volume 9886," Proc. SPIE 9886, Micro-Structured and Specialty Optical Fibres IV, 988601 (19 September 2016); doi:

10.1117/12.2244112

SPIE. Event: SPIE Photonics Europe, 2016, Brussels, Belgium 


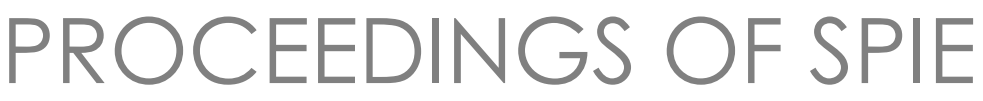

\title{
Micro-Structured and Specialty Optical Fibres IV
}

\author{
Kyriacos Kalli \\ Alexis Mendez \\ Editors \\ 4-6 April 2016 \\ Brussels, Belgium
}

Sponsored by

SPIE

Cosponsored by

B-PHOT-Brussels Photonics Team (Belgium)

Research Foundation Flanders (Belgium)

Visit Brussels (Belgium)

Cooperating Organisations

Photonics 21 (Germany)

EOS-European Optical Society (Germany)

KTN-the Knowledge Transfer Network (United Kingdom)

Graphene Flagship (Belgium)

Photonics Public Private Partnership (Belgium)

Published by

SPIE

\section{Volume 9886}


The papers in this volume were part of the technical conference cited on the cover and title page. Papers were selected and subject to review by the editors and conference program committee. Some conference presentations may not be available for publication. Additional papers and presentation recordings may be available online in the SPIE Digital Library at SPIEDigitallibrary.org.

The papers reflect the work and thoughts of the authors and are published herein as submitted. The publisher is not responsible for the validity of the information or for any outcomes resulting from reliance thereon.

Please use the following format to cite material from these proceedings:

Author(s), "Title of Paper," in Micro-Structured and Specialty Optical Fibres IV, edited by Kyriacos Kalli, Alexis Mendez, Proceedings of SPIE Vol. 9886 (SPIE, Bellingham, WA, 2016) Six-digit Article CID Number.

ISSN: 0277-786X

ISSN: 1996-756X (electronic)

ISBN: 9781510601314

Published by

SPIE

P.O. Box 10, Bellingham, Washington 98227-0010 USA

Telephone +1 3606763290 (Pacific Time) · Fax +1 3606471445

SPIE.org

Copyright (C) 2016, Society of Photo-Optical Instrumentation Engineers.

Copying of material in this book for internal or personal use, or for the internal or personal use of specific clients, beyond the fair use provisions granted by the U.S. Copyright Law is authorized by SPIE subject to payment of copying fees. The Transactional Reporting Service base fee for this volume is $\$ 18.00$ per article (or portion thereof), which should be paid directly to the Copyright Clearance Center (CCC), 222 Rosewood Drive, Danvers, MA 01923. Payment may also be made electronically through CCC Online at copyright.com. Other copying for republication, resale, advertising or promotion, or any form of systematic or multiple reproduction of any material in this book is prohibited except with permission in writing from the publisher. The CCC fee code is 0277-786X/16/\$18.00.

Printed in the United States of America.

Publication of record for individual papers is online in the SPIE Digital Library.

\section{SPIE. DIGITAL}

Paper Numbering: Proceedings of SPIE follow an e-First publication model. A unique citation identifier (CID) number is assigned to each article at the time of publication. Utilization of CIDs allows articles to be fully citable as soon as they are published online, and connects the same identifier to all online and print versions of the publication. SPIE uses a six-digit CID article numbering system structured as follows:

- The first four digits correspond to the SPIE volume number.

- The last two digits indicate publication order within the volume using a Base 36 numbering system employing both numerals and letters. These two-number sets start with 00, 01, 02, 03, 04, $05,06,07,08,09,0 A, 0 B \ldots$. OZ, followed by 10-1Z, 20-2Z, etc. The CID Number appears on each page of the manuscript. 


\title{
Contents
}

\author{
vii Authors \\ ix Conference Committee
}

\section{ADVANCEMENTS TOWARDS MID-INFRARED OPTICAL FIBRES II}

988607 Characterization of holmium fibers with various concentrations for fiber laser applications around $2.1 \mu \mathrm{m}[9886-6]$

MODELLING AND DESIGN OF SPECIALTY OPTICAL FIBRES

988609 Modelling of thermal effects and gain competition in Yb-doped large mode area photonic crystal fibers (Invited Paper) [9886-23]

9886 OA A numerical study on the importance of non-uniform index modification during femtosecond grating inscription in microstructured optical fibers [9886-24]

9886 OB Optimizing large-pitch fibers for higher average powers [9886-25]

9886 OC Self-steepening and intrapulse Raman scattering for supercontinuum generation in optimized photonic crystal fiber at $1.3 \mu \mathrm{m}$ [9886-26]

9886 OD Propagation in few modes fiber with strongly coupled groups of modes [9886-27]

MULTICORE FIBRE DEVICES

9886 OE Supersensitive sensors based on multicore optical fibres (Invited Paper) [9886-8]

9886 OF Femtosecond laser waveguide and FBG inscription in four-core optical fibre [9886-9]

9886 OG Power coupling in multicore optical fiber tapers utilizing out-cladding ferrofluids [9886-10]

$9886 \mathrm{OH}$ Fibre Bragg gratings fabrication in four core fibres [9886-11]

\section{ADVANCED FIBRE SENSORS AND COMPONENTS}

9886 ol Light coupling and routing using a microsphere attached on the endface of a microstructured optical fiber (Invited Paper) [9886-13]

9886 0J Beam-shaping via femtosecond laser-modified optical fibre end faces [9886-14] 
$9886 \mathrm{OL}$ Theoretical investigation into the optimized design of a durable OFSPR hydrogen sensor based on a PdY alloy (Best Student Paper) [9886-16]

\section{INNOVATIVE SILICA AND POLYMER FIBRE SENSORS}

9886 ON Embedding silica and polymer fibre Bragg gratings (FBG) in plastic 3D-printed sensing patches [9886-18]

988600 Comparative study of multimode CYTOP graded index and single-mode silica fibre Bragg grating array for the mode shape capturing of a free-free metal beam [9886-19]

9886 OP Stress tuneable phase shifts of femtosecond-laser microstructured FBG for indentation measurements of biological tissue: experimental and theoretical investigation [9886-20]

9886 OR Ultraviolet inscription of long period gratings on polymer functionalized optical microfibers [9886-22]

\section{POLYMER FIBRE SENSORS}

9886 OS Microstructured polymer optical fibre sensors for opto-acoustic endoscopy [9886-28]

9886 OU Study of thermal annealing effect on Bragg gratings photo-inscribed in step-index polymer optical fibers [9886-30]

9886 OV Annealing effects on strain and stress sensitivity of polymer optical fibre based sensors [9886-31]

9886 OW Fuel level sensor based on polymer optical fiber Bragg gratings for aircraft applications [9886-32]

\section{CHARACTERISATION METHODS APPLICABLE TO SPECIALTY FIBRES}

9886 0X Detailed study of macrobending effects in a wide transmission bandwidth hollow-core photonic bandgap fiber [9886-33]

9886 OY Analysis of spectral light guidance in specialty fibers [9886-34]

$98860 Z$ Atomic-scale imaging of dopant atoms and clusters in Yb-doped optical fibers [9886-35]

\section{SPECIALTY FIBRE PROCESSING AND ADVANCES IN FABRICATION I}

988613 Design and realisation of leakage channel fibres by the powder-in-tube method [9886-39]

988614 Progress in the fabrication of optical fibers by the sol-gel-based granulated silica method [9886-40]

988615 First realization of a birefringent flat-top single-mode fiber [9886-41] 
988617 Dual hollow-core anti-resonant fibres [9886-43]

988619 Creation of a microstructured polymer optical fiber with UV Bragg grating inscription for the detection of extensions at temperatures up to $125^{\circ} \mathrm{C}$ [9886-45]

POSTER SESSION

9886 1 A Self-similar mode of optical pulse propagation in a medium with non-instantaneous multi-photon absorption [9886-46]

9886 1B Spectral properties of optical pulse, containing a few cycles, reflected from or passed through disordered layered structure [9886-47]

9886 1C Suspended-core silica and lead-silicate fibers for nonlinear application and sensing purposes [9886-48]

9886 IF Performance analysis of polymer optical fibre based Fabry-Perot sensor formed by two uniform Bragg gratings [9886-51]

9886 1G Measurement of refractive index profile of non-symmetric, complex silica preforms with high refractive index differences [9886-52]

$98861 \mathrm{H} \quad$ A microsphere-taper cascading structured microfiber for temperature sensing [9886-53]

$98861 \mathrm{~J} \quad$ Active fiber polymer cladding temperature measurement under conditions of laser generation and amplification [9886-55]

$98861 \mathrm{~K}$ Determination of the radial profile of the photoelastic coefficient of polymer optical fibers [9886-56]

$98861 \mathrm{M} \quad$ Numerical analysis of gain and noise figure in different configurations of single-pass and double-pass EDFA [9886-59]

$98861 \mathrm{~N}$ Two-dimensional refractive index profiling of optical fibers by modified refractive near-field technique [9886-60]

988610 Effects in ultrafast laser micromachining PMMA-based optical fibre grating [9886-61]

$98861 \mathrm{P} \quad$ Long period grating response to gamma radiation [9886-62]

$98861 Q \quad$ Online monitoring of gamma irradiated perfluorinated polymer optical fiber [9886-63] 
Proc. of SPIE Vol. $9886988601-6$

Downloaded From: https://www.spiedigitallibrary.org/conference-proceedings-of-spie on 25 Apr 2023 Terms of Use: https://www.spiedigitallibrary.org/terms-of-use 


\section{Authors}

Numbers in the index correspond to the last two digits of the six-digit citation identifier (CID) article numbering system used in Proceedings of SPIE. The first four digits reflect the volume number. Base 36 numbering is employed for the last two digits and indicates the order of articles within the volume. Numbers start with $00,01,02,03,04,05,06,07,08,09,0 A, 0 B . .0 Z$, followed by 10-1Z, 20-2Z, etc.

Abramski, Krzysztof M., 07

Acheroy, Sophie, $1 \mathrm{~K}$

Ahmad, Redwan, 1C

Amezcua-Correa, Rodrigo, OE

Antonio-Lopez, Enrique, $\mathrm{OE}$

Aubrecht, Jan, 07

Badmos, Abdulyezir, $\mathrm{OH}$

Baghdasaryan, Tigran, $\mathrm{OA}$

Bang, Ole, ON, OV, OW, 19

Baravets, Yauhen, 07

Belz, Mathias, OY

Ben Khalifa, Ameni, OD

Ben Salem, Amine, OD

Benda, Adam, $1 G$

Berger, S., 14

Berghmans, Francis, 0A, $1 \mathrm{~K}$

Bigot, L., 15

Boas, J., 0Z, 14

Bouwmans, G., 15

Bradley, T. D., 0X, 17

Broadway, Christian, OS

Cardoso, Marcos R., 10

Carpintero, Guillermo, OS

Cassez, A., 15

Caucheteur, C., OU

Chen, Xianfeng, 10

Chen, Y., OX, 17

Cherbi, Lynda, OC

Cherif, Rim, OD

Coscelli, Enrico, 09

Cucinotta, Annamaria, 09

Delepine-Lesoille, Sylvie, $1 \mathrm{P}$

Downes, F., OL

Dutz, Franz J., OP

El Sayed, A., 0Z, 14, 1N

Etissa, D., 14

Fasano, Andrea, 19

Feng, Guoying, $1 \mathrm{H}$

Ferhat, Mohamed Lamine, OC

Gallego, Daniel, OS

Geernaert, Thomas, 0A, $1 \mathrm{~K}$

Gouriou, P., 15

Gray, D. R., OX

Habert, R., 15

Hayes, J. R., OX, 17

Honzatko, Pavel, 07

Horak, P., 17

$\mathrm{Hu}, \mathrm{X} ., \mathrm{OU}$

Huber, Heinz P., OP
Hugonnot, E., 15

loannou, Andreas, OF, OJ

Jasion, G. T., OX, 17

Jauregui, Cesar, OB

Jelinek, Michal, 07

Kalinovich, Aleksey A., 1A

Kalli, Kyriacos, OF, OJ, 00

Kasik, Ivan, 07

Kinet, D., OU

Klein, Karl-Friedrich, OY

Komanec, Matej, $1 \mathrm{C}$

Komodromos, M., 00

Konstantinou, Maria Georgia, 01

Kosma, Kyriaki, Ol

Koutsides, Charalambos, OF

Krebber, Katerina, 19, 1Q

Kubecek, Vaclav, 07

Kummer, D., 0Z, 14

Lablonde, Laurent, $1 \mathrm{P}$

Lacraz, Amédée, OF, 0J, 00

Lamela, Horacio, OS

Li, Yuhang, OR

Limpert, Jens, OB

Liv, Chen, 10

Marchi, Gabriele, OP

Margulis, Walter, 01

Markos, Christos, 19

Marques, C. A. F., OV, OW

Martan, Tomas, $1 \mathrm{C}$

Mégret, P., OU

Merken, Patrick, $1 \mathrm{~K}$

Mihai, Laura, 1Q

Milenko, Karolina, 0G, OI

Mou, Chengbo, $\mathrm{OH}$

Mrazek, Jan, $1 \mathrm{G}$

Najafi, H., OZ, 14

Negut, Daniel, 1P, 1Q

Nemecek, Tomas, 1C

Nielsen, Kristian, ON, OV, OW, 19

Numkam, E., OX

Ottevaere, Heidi, $1 \mathrm{~K}$

Parali, Ufuk, IM

Pawliszewska, Maria, 07

Pedan, Eugeniy $\vee_{\text {., }} 1 \mathrm{~B}$

Peterka, Pavel, 07, 1G

Petrovich, M. N., OX, 17

Pilz, Soenke, 0Z, 13, 14, 1N

Pınar, Cahit, $1 \mathrm{M}$

Pissadakis, Stavros, 0G, 01 
Podrazky, Ondrej, $1 G$

Poletti, F., 0X, 17

Poli, Federica, 09

Polis, Michael, OF, OJ, 00

Pospori, Andreas, OS, OV, OW, IF

Probostova, Jana, IG

Prusakov, K. Yu., $1 \mathrm{~J}$

Quiquempois, Y., 15

Raisin, P., 13, 14

Raithel, Philipp, OY

Rasmussen, Henrik K., 19

Richardson, D. J., OX, 17

Romano, Valerio, $0 \mathrm{Z}, 13,14,1 \mathrm{~N}$

Rosa, Lorenzo, 09

Roths, Johannes, OP

Ryabushkin, O. A., $1 \mathrm{~J}$

Ryser, Manuel, 14, 1N

Sáez-Rodríguez, David, ON, OV, OW

Sandoghchi, S. R., 0X, 17

Scheuner, J., 13, 14

Schülzgen, Axel, OE

Scol, F., 15

Selleri, Stefano, 09

Sevigny, B., 15

Slanicka, Jiri, $1 G$

Sobon, Grzegorz, 07

Sotor, Jaroslaw, 07

Sporea, Dan, 1P, 1Q

Stajanca, Pavol, 19, 1Q

Stassis, A., 00

Stefani, Alessio, 19

Stephan, Valentin, OP

Stăncalie, Andrei, IP

Stutzki, Fabian, OB

Sugden, Kate, ON, OS

Sun, Qizhen, $\mathrm{OH}$

Sun, Zhongyuan, $\mathrm{OH}$

Suslov, Dmytro, 1C

Sypin, V. E., $1 \mathrm{~J}$

Tagoudi, Eirini, OG

Taylor, C. M., OL

Theodosiou, Antreas, OF, 0J, 00

Thienpont, Hugo, OA, $1 \mathrm{~K}$

Trofimov, Vyacheslav A., 1A, 1B

Tünnermann, Andreas, OB

Valentin, C., 15

Van Newkirk, Amy, OE

Vanvincq, O., 15

Villatoro, Joel, $\mathrm{OE}$

Wang, Changle, $\mathrm{OH}$

Wang, Lijun, OR

Webb, David J., ON, OS, OV, OW, IF, 10

Wheeler, N. V., OX, 17

Woyessa, Getinet, 19

Xian, Pei, $1 \mathrm{H}$

$\mathrm{XU}$, Zhongyang, OR

Yan, Zhijun, $\mathrm{OH}$

Zakharova, Irina G., 1A

Zghal, Mourad, OD

Zhang, Junxi, $\mathrm{OH}$
Zhang, Lin, $\mathrm{OH}$

Zhang, Wei, 10

Zhou, Shouhuan, $1 \mathrm{H}$

Zimmer, Arne Wilhelm, OY

Zubel, Michal G., ON, OS, OV

Zubia, Joseba, OE

Zvanovec, Stanislav, $1 \mathrm{C}$ 


\section{Conference Committee}

Symposium Chairs

Francis Berghmans, Vrijie Universiteit Brussel (Belgium)

Jürgen Popp, Institut für Photonische Technologien e.V. (Germany)

Ronan Burgess, European Commission Photonics Unit (Belgium)

Peter Hartmann, SCHOTT, AG (Germany)

Honorary Symposium Chair

Hugo Thienpont, Vrijie Universiteit Brussel (Belgium)

Conference Chairs

Kyriacos Kalli, Cyprus University of Technology (Cyprus)

Alexis Mendez, MCH Engineering LLC (United States)

Conference Programme Committee

Jean-Luc Adam, Université de Rennes 1 (France)

Jean-Louis Auguste, XLIM Institut de Recherche (France)

Ole Bang, Technical University of Denmark (Denmark)

Neil G. R. Broderick, The University of Auckland (New Zealand)

Benoit Cadier, iXFiber SAS (France)

Adrian L. Carter, Nufern (United States)

Liang Dong, Center for Optical Materials Science + Engineering Technologies (United States)

Henry H. Du, Stevens Institute of Technology (United States)

Sebastien Fevrier, XLIM Institut de Recherche (France)

Jiri Kanka, Institute of Photonics and Electronics of the ASCR, v.v.i.

(Czech Republic)

Karl-Friedrich Klein, Technische Hochschule Mittelhessen (Germany)

Jonathan C. Knight, University of Bath (United Kingdom)

Michael Komodromos, Frederick University (Cyprus)

Walter Margulis, Acreo Swedish ICT AB (Sweden)

Saeed Rehman, Fibertronix AB (Sweden)

Valerio Romano, Bern University of Applied Sciences (Switzerland)

Kunimasa Saitoh, Hokkaido University (Japan)

Kay Schuster, Institut für Photonische Technologien e.V. (Germany)

Sergei V. Semyonov, Fiber Optics Research Center

(Russian Federation)

Waclaw Urbanczyk, Wroclaw University of Technology (Poland)

David J. Webb, Aston University (United Kingdom) 
Alexei M. Zheltikov, Lomonosov Moscow State University (Russian Federation)

Hwa-Yaw Tam, The Hong Kong Polytechnic University (Hong Kong, China)

\section{Session Chairs}

1 Advancements Towards Mid-Infrared Optical Fibres I

Kyriacos Kalli, Cyprus University of Technology (Cyprus)

2 Advancements Towards Mid-Infrared Optical Fibres II

Kyriacos Kalli, Cyprus University of Technology (Cyprus)

3 Modelling and Design of Specialty Optical Fibres

David J. Webb, Aston University (United Kingdom)

$4 \quad$ Multicore Fibre Devices

Kyriacos Kalli, Cyprus University of Technology (Cyprus)

5 Advanced Fibre Sensors and Components

Kyriacos Kalli, Cyprus University of Technology (Cyprus)

6 Innovative Silica and Polymer Fibre Sensors

David J. Webb, Aston University (United Kingdom)

$7 \quad$ Polymer Fibre Sensors

Karl-Friedrich Klein, Technische Hochschule Mittelhessen (Germany)

8 Characterisation Methods Applicable to Specialty Fibres Manuel Ryser, Universität Bern (Switzerland)

9 Specialty Fibre Processing and Advances in Fabrication I Kyriacos Kalli, Cyprus University of Technology (Cyprus)

10 Specialty Fibre Processing and Advances in Fabrication II Kyriacos Kalli, Cyprus University of Technology (Cyprus) 